\title{
The Effect of Luminance Contrast Between Sign and Surrounding Object on Gaze Behavior: A Study in Virtual Metro Station Environment with Rendered Static/Dynamic Panorama
}

\author{
Xiaoqun Ai \\ College of Mechanical Engineering and Automation, Huaqiao University, Xiamen, China \\ College of Architecture, The Central Academy of Fine Arts, Beijing, China \\ E-mail: axq777@gmail.com
}

Yufei Liu, Zhendong $\mathrm{Wu}^{1}$, Jingchun Chai, Xinping Ju,Wenxiang Duan,Lintao Zhao and Ying Liang

College of Mechanical Engineering and Automation, Huaqiao University, Xiamen, China

E-mail: 974788863@qq.com,wudesigner@gmail.com, chensson@qq.com,1026167399@qq.com,wx-duan@qq.com, 875370872@qq.com,1092943764@qq.com

Keywords: immersive virtual reality, eye tracking, lighting simulation, luminance contrast, sign, fixation

Received: July 26, 2021

\begin{abstract}
This paper investigated the effect of luminance contrast between sign and surrounding object on the gaze behavior of pedestrian, static or dynamic $360^{\circ}$ panorama rendered in a virtual environment applied to simulate the wayfinding of pedestrians in the metro stations. Fifty-five participants observed the sign posters and the advertisement boards with distinct luminance contrasts (31 of them were in static levels, 24 of the rest were in dynamic levels) and were asked to point out the graphic or textual changes in the area after 30s. The eye tracker recorded ocular data, and glare perception was inquired by questionnaire. The result of T-test and Regression analysis revealed that luminance contrast was a saliency feature distinguishing visual targets and surrounding objects. The correlation between the value of luminance contrast and fixations, fixation durations on the sign is negative. Each increase in luminance contrast by one unit reduces the mean of fixations by 0.826, accompanied by a stronger feeling of glare, which indicated the strategic adaption of visual attention. The study contributed to our understanding of a new sight of lighting design in public traffic places and confirmed that lighting simulation in an immersive virtual environment can effectively analyzes visual perception.
\end{abstract}

Povzetek: Analizirana je prepoznavnost znakov v okolju podzemne železnice.

\section{Introduction}

\subsection{Lighting in metro station}

LED is widely used in metro stations illuminated artificially with higher luminous efficiency and lower cost. There are various luminous objects (as shown in Figure 1), some of them are ceiling lights that provide a basic lighting environment, some are luminous graphs that provide navigation or commercial information, others are road signs scattered in the metro station, forming dazzling visual cues in the eye of pedestrian (as shown in Figure 2). All of these cause difficulties for wayfinding. Sign poster refers to road signs and luminous graphs with navigation information, which is critical for wayfinding. To guide pedestrians reach their destinations faster, sign poster is expected to play a leading role in the vision of pedestrian, it can be achieved by reasonably controlling the relationship of luminance in various luminous objects.

\subsection{Luminance contrast and visual attention}

The national lighting standards of America, Germany, Japan, and China make the minimum request based on illuminance for indoor public places such as the metro station, which tends to fit the lighting system's requirements rather than refining pedestrian's visual effect [1]. Luminance emphasizes light reaching the viewer's position, and it is the only photometric indicator directly related to visual perception [2]. Improving the contrast of task attributes can improve task performance without raising illuminance [3]. Several researchers have explored how to transform the visual stimuli into specific luminance and luminance contrast indicators $[4,5,6]$.

Pedestrians search the targets to find their way, who constantly and swiftly divert visual attention to new local areas with high frequency until the valuable information for navigation has been captured. Then, the brain centralizes limited cognitive resources to prioritize them and ignores other information. The bottom-up

\footnotetext{
${ }^{1}$ Corresponding author
} 
mechanisms show that luminance and luminance contrast guide pedestrians' visual behavior and identify visual search results as a saliency feature [7]. In this process,

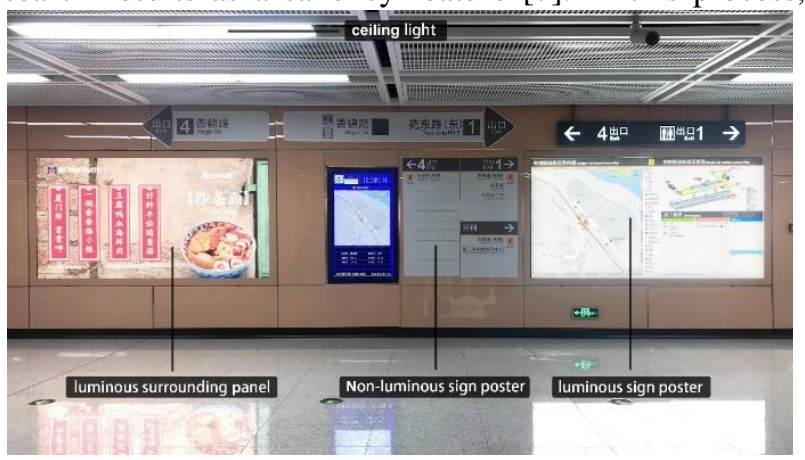

Fig 1: The types of light sources in metro station.

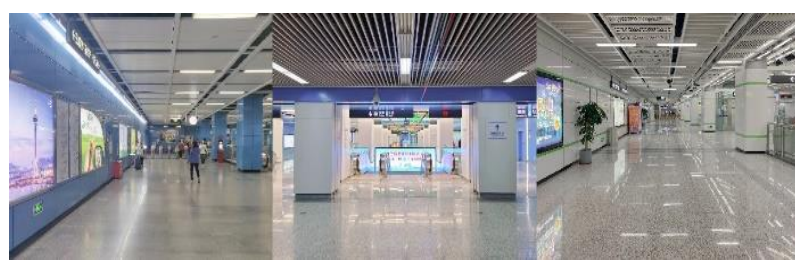

Fig 2: The existing state of lighting in Chinese metro stations.

information is input quickly and unconsciously, only after that, the top-down cognitive regulation will gradually function [8].

There are strict regulations for the lighting of sign. However, its surrounding area's luminance varies a lot due to the change of the objects next to it, such as replacing advertising boards with different luminance regularly, adding vending machines with glowing screens, and laying materials with various lighting attributes on the wall, etc. Luminance contrast influences visual behavior as one of the visual saliency features, previous studies have ignored its changes and effects. Therefore, we are supposed to highlight the advantages of signs against other light sources in pedestrians' eyes and make the navigation information easier to attract pedestrians' attention. By eye-tracking technology, the luminance contrast characteristics that benefit pedestrians' observations can be estimated quantitatively via ocular data such as fixations and fixation durations [9,10,11]. Besides, measurements for subjective lighting perception are included, as endogenous factors like cognitive load also influence the process of attention [12].

Overall, the present study was designed to determine the effect of the luminance contrast between sign and surrounding objects on gaze behavior and pedestrians' lighting perception, expecting to summarize the appropriate lighting control strategies and provide empirical evidence for relevant lighting research and standards. The objectives of this study were to answer the following research questions:

- Does luminance contrast between sign and surrounding object influence the gaze behavior of pedestrian?
- How does luminance contrast between sign and surrounding object influence the gaze behavior of pedestrian?

How does luminance contrast between sign and surrounding object influence the gaze behavior of pedestrian?

\subsection{Visual reality and lighting simulation}

In the real world, it is hard to control all the variables in the physical setting to examine the effect of specific conditions, especially in a metro station that is usually complex and crowded. Virtual reality technology gives the possibility to collect data in a flexible, timely and costeffective way with high accuracy of experimental control, and the replicable experiments can be performed without any loss $[13,14,15]$.

In all virtual environments, $360^{\circ}$ panorama is considered a promising media to replace physical lighting environments. Compared with the immersive virtual environment rendered in real-time, the advanced rendering in a whole field of vision offers the most realistic visual effects with mature $3 \mathrm{D}$ graphics software, while lighting conditions are under accurate control by professional lighting software. The use of head-mounted displays makes visual perception broader and more accessible, also avoids loss in the spread of light. The experiment of Schrom-Feiertag and Settgast confirmed the usability of an assessment system combining eye tracking with the virtual environment, which has shown that this method allows for the creation of attention maps and the identification of objects of interest based on eyetracking. A similar methodology is widely used in interior lighting for occupants along with road lighting for drivers and pedestrians $[16,17,18,19]$.

\section{Method}

\subsection{Environment and apparatus}

\subsubsection{Physical lighting environment}

We investigated several stations in Xiamen Metro Line 1 in China to pick up photography's representative locations. The selected spots were sited in straight corridors with obvious walking direction and decision points where the side walls showed massive signs and advertisement information that are not related to wayfinding, the luminance attributes of this information were uneven, making discomfort glare obvious in pedestrians' eye.

The camera was Canon EOS 5D Mark II full-frame camera outfitted with Sanyo 8mm F3.5 Circular Fisheye lens, the aperture to $f / 5.6$, the shutter speed to $1 / 40 \mathrm{~s}$, the sensitivity to IOS 640, and the camera was placed at the height of $1.75 \mathrm{~m}$ to take photographs in front and behind, which represented the view from the eye, [20,21,22] as shown in Figure 3.

A measurement grid is often established to cover the space's overall lighting [2]. Most current standards recommend the ground as the lighting measurement and calculation plane [23]. Pedersen and Johansson [24] 

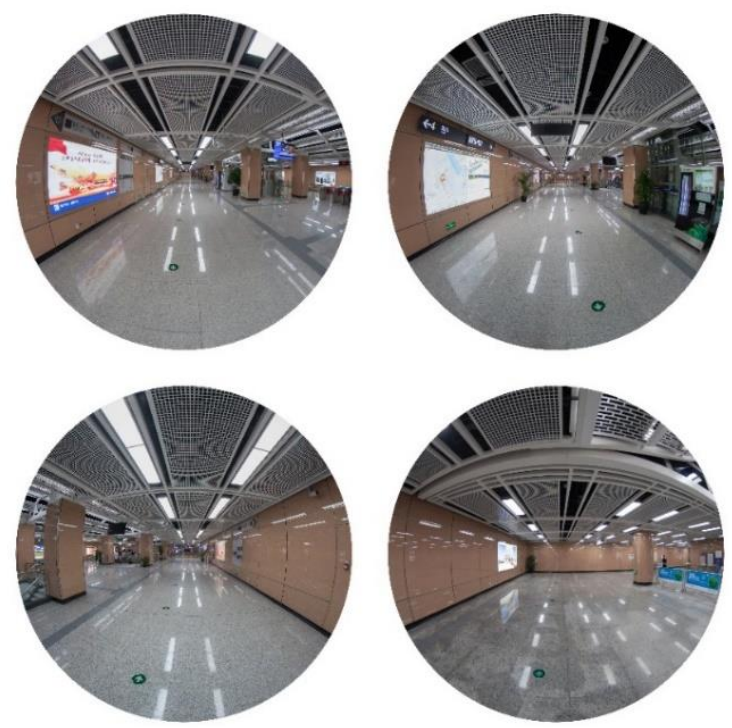

Fig 3: Photographs of selected corridors.
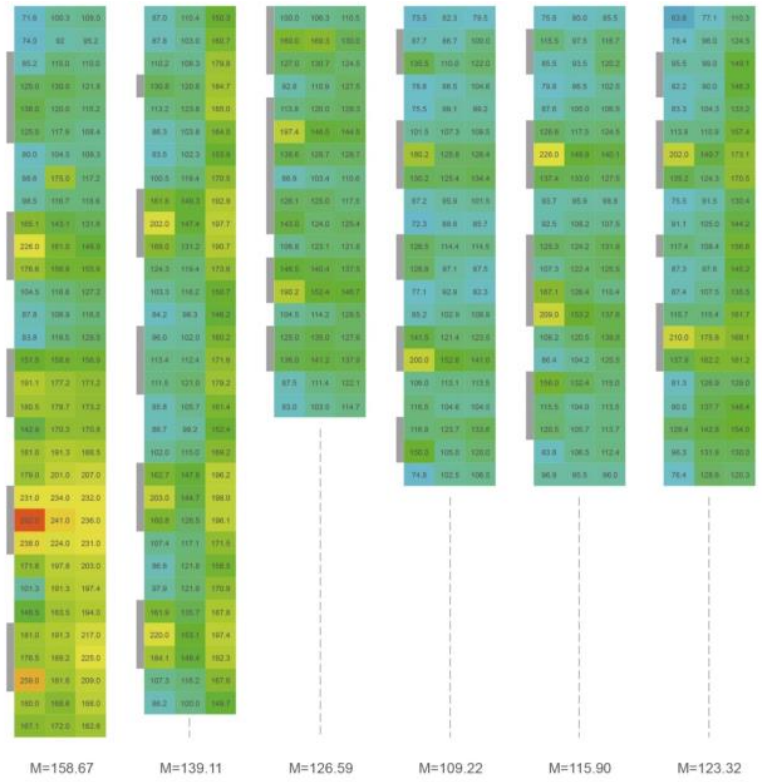

Fig 4: Illuminance (lx) of selected corridors.
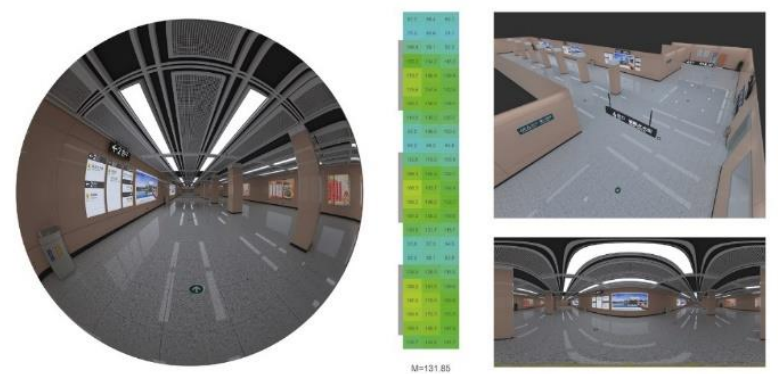

Fig 5: Virtual lighting environment with illuminance (lx).

measured the illuminance for each grid point on-road and calculated the average value. Kim et al. [25] measured the illuminance value for 25 uniform points horizontally distributed on the ground and expressed them in a grid chart. Referring to the above studies, we made several grid measurements on the ground next to the wall on selected spots, containing the lighting information of ceiling lights, advertisement board, and road signs to the greatest extent. Six areas shown in Figure 4 were measured with a portable illuminance meter (PEAKMETER PM6612L digital illuminance meter, minimum measurement unit: $0.1 \mathrm{~lx}$ ), the size of a single cell was $0.8 \times 1.2 \mathrm{~m}$, the acreages of the whole grid ranged from $17.6 \mathrm{~mm}^{2}$ to $81.92 \mathrm{~mm}^{2}$, the grey blocks represented where the luminous graphs were.

\subsubsection{Virtual lighting environment}

According to the photos, a 1:1 full-scale metro station model was built in 3Ds Max. The corridor's length was extended so that there were three fields of view with the same content and distance in the scene to meet the experiment's needs. A virtual camera with identical shooting parameters is placed at the same position as the corridor extended forward. The lighting analysis tools VRayLightMeter based on V-Ray renderer were used for high-precision visualizations of the lighting environment. VRayLightMeter was established on the ground with the same position and width division as the measurement grid, and the length division takes as the average of the six measured grids. According to Jones and Reinhart [20], the simulation error between physical lighting environment and virtual lighting environment based on images can be calculated by the relative error of mean luminance in two areas. Constrained by the spot, the simulation error represents the relative error of mean illuminance in measured areas. Assume that the first measured area is named as "A", the points distributed there are named as " $a$ ", the number of the points is " $n_{a}$ ", secondly, the next measured area is named as " $\mathrm{B}$ ", the points distributed there are named as " $b$ ", the number of the points is " $n_{b}$ " and so on to the last measured area $\mathrm{M}$, the mean illuminance is $\bar{E}$ and the simulation error is $\eta$, the formula can be defined as:

$$
\bar{E}=\frac{\Sigma E_{a}+\Sigma E_{b}+\cdots+\Sigma E_{m}}{n_{a}+n_{b}+\cdots+n_{m}}
$$

$$
\eta=\frac{\bar{E}_{\text {simu }}-\bar{E}_{\text {real }}}{\bar{E}_{\text {real }}} \times 100 \%
$$

Groups controlled the light sources, and all the ceiling lights divide into one group, the sign posters and the advertisement boards on the wall were divided into another group, the luminance of the light sources in one group stayed consistent. We adjusted the luminance of light sources of these two groups by illuminance distribution and renderings until the simulation error decreased to the illuminance of the false panel kept up with the real ground, by then, $\bar{E}_{\text {simu }}=131.85 \mathrm{~lx}, \eta=0 \%$, the initial virtual lighting environment is shown in Figure 5.

$\eta=0 \%$ did not mean error-free, as there were inevitable human and system errors involved in the simulation. At the stage of modeling, the corridor was duplicated and extended to meet the experiment's needs. 
Hence the illuminance distribution was different from a real place. In the physical world, light entered our eyes through a series of refraction and reflection, but the light from the screen of HMD was directly cast to our eyes, resulting in the viewer constantly overestimated the brightness of scenes [26]. To avoid this, we lowered the virtual camera' exposure to an appropriate degree by observation in HMD [27].

\subsubsection{Integration in apparatus}

The panoramas of each field of view and the corridor's entrance were rendered then imported into Tobii Pro Lab software in order. The switch key was set to present all the viewpoints sequentially, imitating the process of pedestrians' walking on the way [28]. HTC VIVE Pro Eye, a head-mounted display integrating Eye tracking system was applied, resolution of monocular display was $1440 \times$ $1600 \mathrm{px}$, Eye tracker sampled with the rate of $120 \mathrm{~Hz}$, both parties were connected with the computer through a HDMI cable, gaze data was recorded and generated in Tobii Pro Lab. Experimental setting in virtual environment is shown in Figure 6. The Tobii I-VT filter recognized eye movements based on the ocular velocity classification algorithm.

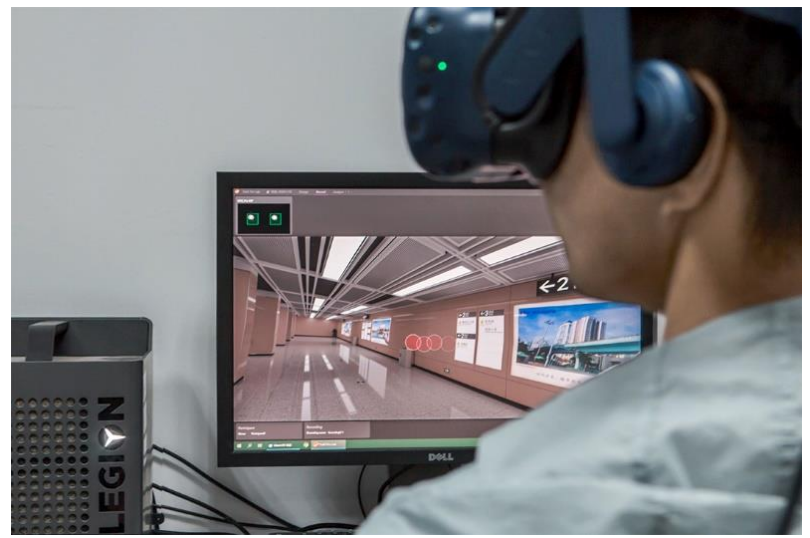

Fig 6: Experimental setting in virtual environment.

\subsection{Experiment design}

According to the research questions previously defined, and based on the literature review and technical method, we formulated a series of research hypotheses to verify the relation between gaze behavior of pedestrians and luminance contrast in the virtual environment. The hypotheses are detailed in Table 1.

\subsubsection{Independent variable: luminance contrast}

The luminance contrast between sign poster and advertisement board was taken as an independent variable. We set the luminance value of the sign poster $\left(L_{S}\right)$ to the minimum $\left(L_{S}=0\right)$ and compensatorily adjusted the luminance value of the advertisement board $\left(L_{A}\right)$ within the constant scope of simulation error $\left(\bar{E}_{\text {simu }}=131.85 \mathrm{~lx}\right.$, $\eta=0 \%)$, while the rest of the light sources remained unchanged. Reversely, set the luminance value of the advertisement board to the minimum $\left(L_{A}=0\right)$ then compensatorily adjusted the luminance value of the sign poster. In this way, two static levels were created. Two dynamic levels were composed with uniform transitions from starting state (initial lighting environment) to ending state (other static levels) to analyze variety.

\begin{tabular}{|c|c|c|}
\hline & $\begin{array}{l}\text { Static luminance } \\
\text { contrast }\end{array}$ & $\begin{array}{l}\text { Dynamic } \\
\text { luminance contrast }\end{array}$ \\
\hline fixations & $\begin{array}{l}\text { H1 - Numbers of } \\
\text { fixations on sign } \\
\text { posters will be } \\
\text { different in } \\
\text { condition with or } \\
\text { without luminance } \\
\text { contrast between } \\
\text { sign posters and } \\
\text { advertisement } \\
\text { board. }\end{array}$ & $\begin{array}{l}\text { H4 - Numbers of } \\
\text { fixations on sign } \\
\text { posters will change } \\
\text { with the value of } \\
\text { luminance contrast } \\
\text { between sign } \\
\text { posters and } \\
\text { advertisement } \\
\text { board. }\end{array}$ \\
\hline $\begin{array}{l}\text { Fixation } \\
\text { durations }\end{array}$ & $\begin{array}{l}\text { H2 - Durations of } \\
\text { fixations on sign } \\
\text { posters will be } \\
\text { different in } \\
\text { condition with or } \\
\text { without luminance } \\
\text { contrast between } \\
\text { sign posters and } \\
\text { advertisement } \\
\text { board. }\end{array}$ & $\begin{array}{l}\text { H5 - Durations of } \\
\text { fixations on sign } \\
\text { posters will change } \\
\text { with the value of } \\
\text { luminance contrast } \\
\text { between sign } \\
\text { posters and } \\
\text { advertisement } \\
\text { board. }\end{array}$ \\
\hline $\begin{array}{l}\text { Glare } \\
\text { rating }\end{array}$ & $\begin{array}{l}\text { H3 - Glare ratings } \\
\text { will be different in } \\
\text { condition with or } \\
\text { without luminance } \\
\text { contrast between } \\
\text { sign posters and } \\
\text { advertisement } \\
\text { board. }\end{array}$ & \\
\hline
\end{tabular}

Table 1: Experiment hypotheses.

The luminance of the task area describes the luminance contrast due to luminance difference of gaze area, luminance of surrounding area, max luminance of the area, minimum luminance of the area, and mean luminance of the area. A ubiquitously applicable metric for all lighting conditions has not been established to date $[4,20,0]$. According to the study's needs, the luminance contrast was defined as:

$$
R=\frac{L_{H}}{L_{L}}
$$

$L_{H}$ is the mean luminance of the area, which is the brighter one of the task area and surrounding area. $L_{L}$ is the mean luminance of the area, which is the darker one of the two, that means when the sign poster is brighter, $R=$ $\frac{L_{S}}{L_{A}}$, conversely, when the advertising board is brighter, $R=$ $\frac{L_{A}}{L_{S}}$. If the sign poster's luminance is consistent with the luminance of the advertising board, there is no luminance difference, the value of luminance contrast reaches to the 


\begin{tabular}{|c|c|c|c|c|}
\hline Level type & Diagram & Illustration & Luminance & Diagram \\
\hline \multirow{2}{*}{ Static level } & $=0$ & $\begin{array}{l}\text { The initial lighting } \\
\text { environment, the luminance } \\
\text { of the sign poster and the } \\
\text { advertising board was } \\
\text { consistent. }\end{array}$ & $\begin{array}{l}L_{S}=126.5 \\
L_{A}=126.5\end{array}$ & $\begin{array}{l}\text { Without luminance } \\
\text { contrast }\end{array}$ \\
\hline & 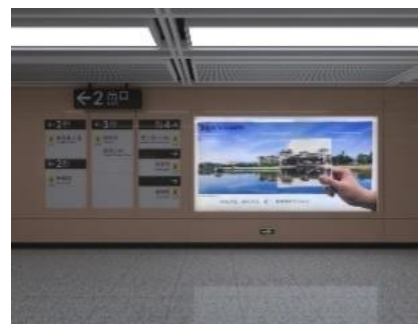 & $\begin{array}{l}\text { The luminance of the sign } \\
\text { poster was set to minimum, } \\
\text { the luminance of the } \\
\text { advertising board was } \\
\text { compensatorily adjusted } \\
\text { within the scope of } \\
\text { simulation error. }\end{array}$ & $\begin{array}{l}L_{S}=0.0 \\
L_{A}=157.4\end{array}$ & $\begin{array}{l}\text { With luminance } \\
\text { contrast }\end{array}$ \\
\hline \multirow{3}{*}{$\begin{array}{l}\text { Dynamic } \\
\text { level }\end{array}$} & mand & $\begin{array}{l}\text { The luminance of the } \\
\text { advertising board was set to } \\
\text { minimum, the luminance of } \\
\text { the sign poster was } \\
\text { compensatorily adjusted } \\
\text { within the scope of } \\
\text { simulation error. }\end{array}$ & $\begin{array}{l}L_{S}=310.1 \\
L_{A}=0.0\end{array}$ & $\begin{array}{l}\text { With luminance } \\
\text { contrast }\end{array}$ \\
\hline & & $\begin{array}{l}\text { The luminance of the sign } \\
\text { poster varied from medium } \\
\text { to maximum while the } \\
\text { luminance of the advertising } \\
\text { board varied from medium } \\
\text { to minimum }\end{array}$ & $\begin{array}{l}L_{S}=126.5 \\
L_{A}=126.5 \\
\text { to } \\
L_{S}=310.1 \\
L_{A}=0.0\end{array}$ & $\begin{array}{lr}\text { Luminance contrast } \\
\text { varied } & \text { from } \\
\text { minimum } & \text { to } \\
\text { maximum } & \end{array}$ \\
\hline & & $\begin{array}{l}\text { The luminance of the sign } \\
\text { poster varied from medium } \\
\text { to minimum value while the } \\
\text { luminance of the advertising } \\
\text { board varied from medium } \\
\text { to maximum value }\end{array}$ & $\begin{array}{l}L_{S}=126.5 \\
L_{A}=126.5 \\
\text { to } \\
L_{S}=0.0 \\
L_{A}=157.4\end{array}$ & $\begin{array}{lr}\text { Luminance } & \text { contrast } \\
\text { varied } & \text { from } \\
\text { minimum } & \text { to } \\
\text { maximum } & \end{array}$ \\
\hline
\end{tabular}

Table 2: Luminance contrast levels.

minimum $(R=1)$. It is generally suggested that the luminance ratio of the task area and surrounding area should be maintained in the value of 3, otherwise, there would be visual discomfort or glare. Hence the reasonable value of luminance contrast should be kept in the range of $[1,2,3,20]$. The static and dynamic luminance contrast levels are shown in Table 2, what needs to be more specifically noticed is the static levels represent the states of luminance contrast, while the dynamic levels represent the extent of luminance contrast, only in the latter the value of luminance contrast makes sense.

According to the definition of luminance contrast, we calculated its values at every $3 \mathrm{~s}$ interval during the observation period of $0-30 \mathrm{~s}$, which different measurement levels as shown in Table 3. Taking the stable state of participants and the reasonable range of luminance contrast into account when they completed the task, only the data within 3-27s was adopted.

\subsubsection{Dependent variable: visual attention and lighting perception}

Visual attention - The typical performance of visual attention, i.e., diversion and concentration, correspond to different ocular data metrics. We cared about the effect of luminance contrast on the pedestrians' observation of the sign, so the area where the sign poster was placed was marked as the area of interest. The number of fixations and their total durations in the area of interest was collected, the former was expressed by $F_{n}$ while the latter was written as $F_{d}[4,30]$.

To make the visual attention more focalized, a visual task was assigned, requiring participants to point out the graphic or textual changes on the sign poster or advertisement board, which evolved from the classic attention paradigm, the visual search task. The changes 


\begin{tabular}{|c|c|c|l|l|}
\hline Measurement level & $\begin{array}{c}\text { Time } \\
\text { Unit: } \mathrm{s}\end{array}$ & $\begin{array}{c}L_{H} \\
\text { Unit: } \mathrm{cd} / \mathrm{m}^{2}\end{array}$ & $\begin{array}{c}L_{L} \\
\text { Unit: } \mathrm{cd} / \mathrm{m}^{2}\end{array}$ & $\mathrm{R}$ \\
\hline- & start & 156.55 & 156.55 & $\mathrm{~min}$ \\
\hline 1 & 3 & 156.64 & 140.90 & 1.11 \\
\hline 2 & 6 & 156.72 & 125.24 & 1.25 \\
\hline 3 & 9 & 156.81 & 109.59 & 1.43 \\
\hline 4 & 12 & 156.89 & 93.93 & 1.67 \\
\hline 5 & 15 & 156.98 & 78.28 & 2.01 \\
\hline 6 & 18 & 157.06 & 62.62 & 2.51 \\
\hline 7 & 21 & 157.15 & 46.97 & 3.35 \\
\hline 8 & 24 & 157.23 & 31.31 & 5.02 \\
\hline- & 27 & 157.32 & 15.66 & 10.05 \\
\hline- & end & 157.40 & 0.00 & max \\
\hline
\end{tabular}

Table 3: Luminance contrast in dynamic levels.

* Time means the seconds in observation period, it is the equidistant time points help to measurement and statistic.

were apparent enough to attract attention, and they appeared randomly on the sign poster or advertisement board. The participants were given the 30 s for observation before the search period. During the observation period, the ocular data were recorded while the visual search task has not finished. It would arouse the target searching awareness of participants but not affect the actual performance of visual attention. The contents of the task are shown in Table 4.

In static levels, the measurement time was counted starting from the first fixation in the area of interest during the whole observation period of the $30 \mathrm{~s}$. In dynamic levels, the measurement time was divided into intervals of every three seconds to obtain fixations corresponding to different luminance contrast values in each period [31].

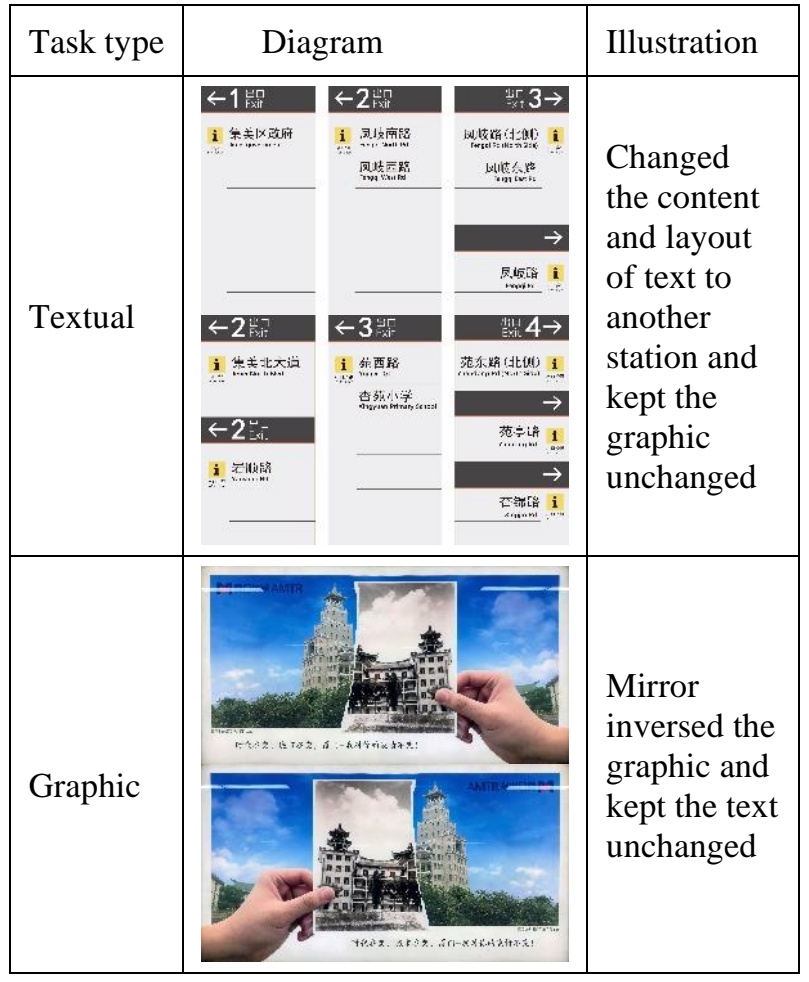

Table 4: The content of visual task.
Lighting perception - The de Boer rating scale for discomfort glare was applied to verify the effect of luminance contrast in lighting perception [32,33]. The participants' perception of the lighting area was queried during the post-task phase. Five ratings of 1 to 5 for the scale represented the sense of "unbearable", "disturbing", "acceptable", "satisfactory" and "unnoticeable" in turn. The higher the rating is, the more comfortable and satisfied the viewer felt.

\subsubsection{Experiment design}

There were two sections in our experiment, the qualitative one and the quantitative one. Qualitative experiments mainly explored whether the luminance contrast would take effect on pedestrians. This section hypothesized that visual attention and lighting perception on the condition with luminance contrast would be different from the one on the condition without luminance contrast, in which the independent variables were static levels, the dependent variables were participants' fixations, fixation durations on the sign poster in an observation period of the 30 s and the glare ratings in the post-task period. Participants were randomly grouped for once measurement of an independent level to eliminate the threat of simulator sickness and maturation effect.

Quantitative experiment mainly researched how the luminance contrast affected pedestrians. The independent variables were the eight values of luminance contrast in dynamic levels, sequentially corresponding to the one in every three seconds during the observation period of 3 to 27Seconds, the dependent variables were participants' fixations and fixation durations in that time, the rest of the settings remained the same as the qualitative experiment.

\subsection{Participants}

In this study, 55 participants were recruited of which 38 were male $(69.10 \%)$, and 17 were female $(30.90 \%)$. All of our recruited samples were, ranging from 18 to 28 years $(\mathrm{M}=22.25, \mathrm{SD}=2.83)$. There were no abnormal conditions such as severe myopia and astigmatism in their eyes or apparent simulator sickness during the experiment. Most 
participants were unfamiliar with the metro station and rarely or never had taken Xiamen Metro Line One. 31 observers participated in the qualitative experiment, while 24 observers participated in the quantitative experiment.

\subsection{Procedure}

The participants were given informed consent then filled in their genders, ages, eye conditions, and familiarities with the metro station. They learned in advance with the sign poster and advertisement board presented in VE through slideshows. After that, the participants entered the virtual environment by wearing HMD and browsing at the straight corridor entrance about 5-10s, then carried to the first viewpoint. The researcher instructed viewers to observe the sign poster and advertisement board on the nearest wall for 30 seconds, when timed up, they were asked to turn back. Meanwhile, the content of the target areas was replaced. Afterward, the participants turned around and pointed out the difference. The above process was performed repeatedly twice. The first time was practice and the second time was a formal implement. There was a short interval of time between these two tasks to eliminate the maturation effect's threat. When all the tasks were completed, the rating for discomfort glare was reported verbally.

\section{Results}

The sample size in qualitative and quantitative experiments reached what is required for the large effect size when $\alpha=0.05$. All statistical analyses were performed with the software IBM SPSS v.24 and a significance level of $5 \%$ was considered.

\subsection{Qualitative experiment in static levels}

To test the hypothesis for the visual attention and lighting perception on the condition with luminance contrast would be different from the one on the condition without luminance contrast, a comparison was made between groups in fixations, fixation durations, and glare ratings by Independent-Samples T-test and Mann-Whitney U test.

\subsubsection{Visual attention in luminance contrast}

The data met the assumption of the normality of distribution and equality of variances. The IndependentSamples T-test results revealed significant differences $(\mathrm{t}=-$ 2.983, $\mathrm{df}=28, \mathrm{P}=.006$ ) between the condition with luminance contrast and the condition without luminance contrast in fixations and fixation durations $(\mathrm{t}=-3.438$, $\mathrm{df}=28, \mathrm{P}=.002)$. Compared with the condition without luminance contrast $(\mathrm{M}=59.43, \mathrm{SD}=20.00 ; \mathrm{M}=13.71$, $\mathrm{SD}=4.90)$, Participants' fixations $(\mathrm{M}=40.13, \mathrm{SD}=15.41)$ and fixation durations $(\mathrm{M}=8.02, \mathrm{SD}=4.17)$ on the sign poster were less, as shown in Figure 7.
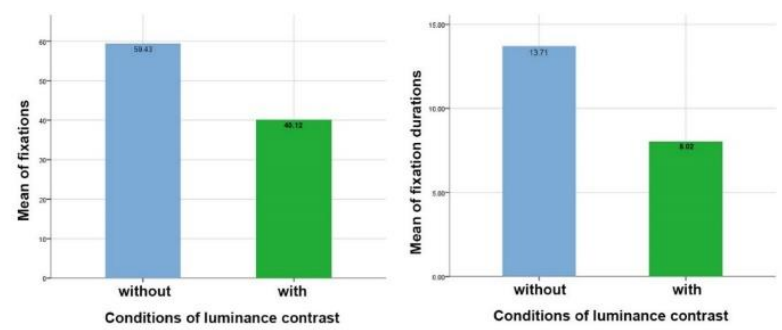

Fig 7: The effect of luminance contrast on fixations and fixation durations on the sign poster.

\subsubsection{Lighting perception in luminance contrast}

The Mann-Whitney $U$ test results suggested that luminance contrast influenced the glare ratings $(\mathrm{U}=-3.067$, $\mathrm{P}=.002$ ). The group participants with luminance contrast gave lower ratings $(\mathrm{M}=2.56, \mathrm{SD}=1.03)$ than the one given by those in the group without luminance contrast $(\mathrm{M}=3.80, \mathrm{SD}=0.94)$, indicating that on the condition with luminance contrast, more severe visual interference participants had perceived. The distribution of ratings between groups is shown in Figure 8.

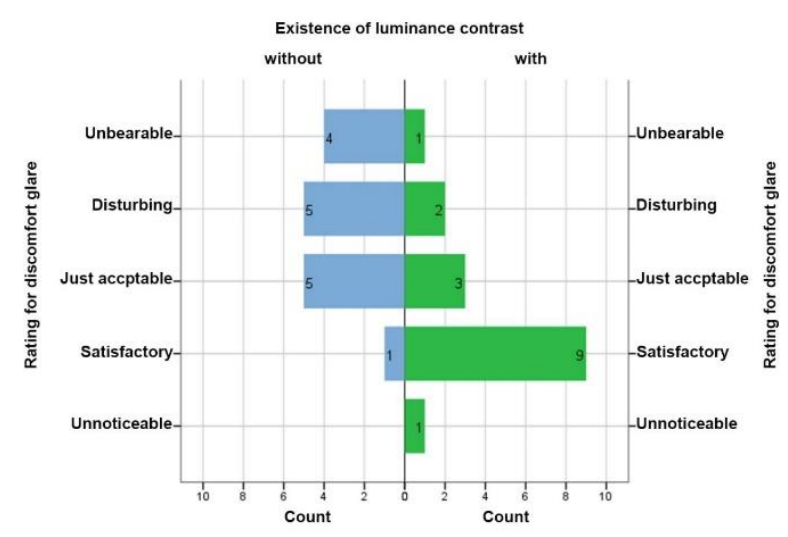

Fig 8: The effect of luminance contrast on glare rating.

\subsection{Quantitative experiment in dynamic levels}

The qualitative experiment's hypothesis had multiple meanings, and the central hypothesis expressed that pedestrians' visual perceptions were different on conditions of different values of luminance contrast. Furthermore, the secondary hypothesis conveyed that pedestrians' visual perceptions vary with the luminance contrast value changes. To test the main hypothesis, comparisons were made for the differences between fixations and fixation durations affected by the values of luminance contrast, one-way repeated measures analyses of variance were run intra groups. To test the secondary hypothesis, Pearson correlations and regression analyses were performed to verify the relations between the values of luminance contrast, fixations and fixation durations on the sign poster. 


\begin{tabular}{|c|c|c|c|c|c|c|c|c|}
\hline & \multirow{2}{*}{ Mean } & \multirow{2}{*}{$\begin{array}{l}\text { Std. } \\
\text { Deviation }\end{array}$} & \multicolumn{3}{|c|}{ Skewness } & \multicolumn{3}{|l|}{ Kurtosis } \\
\hline & & & Statistic & Std. Error & Z-Score & Statistic & Std. Error & Z-Score \\
\hline Fixation_1 & 8.54 & 4.67 & -0.48 & 0.47 & -1.02 & -1.03 & 0.92 & -1.12 \\
\hline Fixation_2 & 7.58 & 3.45 & -0.77 & 0.47 & -1.63 & -0.58 & 0.92 & -0.63 \\
\hline Fixation_3 & 8.13 & 3.38 & -0.63 & 0.47 & -1.33 & -0.17 & 0.92 & -0.19 \\
\hline Fixation_4 & 7.17 & 4.29 & -0.33 & 0.47 & -0.69 & -0.76 & 0.92 & -0.83 \\
\hline Fixation_5 & 5.96 & 4.72 & 0.22 & 0.47 & 0.46 & -1.38 & 0.92 & -1.50 \\
\hline Fixations_6 & 6.00 & 4.43 & -0.32 & 0.47 & -0.68 & -1.50 & 0.92 & -1.63 \\
\hline Fixations_7 & 4.71 & 3.75 & 0.31 & 0.47 & 0.66 & -0.71 & 0.92 & -0.77 \\
\hline Fixation_8 & 5.42 & 3.66 & -0.36 & 0.47 & -0.77 & -1.11 & 0.92 & -1.21 \\
\hline Fixation Duration_1 & 1.35 & 0.77 & -0.51 & 0.47 & -1.09 & -1.13 & 0.92 & -1.23 \\
\hline Fixation Duration_2 & 1.52 & 0.73 & -0.76 & 0.47 & -1.61 & -0.51 & 0.92 & -0.56 \\
\hline Fixation Duration_3 & 1.59 & 0.73 & -0.66 & 0.47 & -1.39 & -0.62 & 0.92 & -0.68 \\
\hline Fixation Duration_4 & 1.45 & 0.85 & -0.59 & 0.47 & -1.24 & -0.96 & 0.92 & -1.05 \\
\hline Fixation Duration_5 & 1.20 & 0.98 & 0.17 & 0.47 & 0.36 & -1.58 & 0.92 & -1.73 \\
\hline Fixation Duration_6 & 1.33 & 1.02 & -0.22 & 0.47 & -0.47 & -1.70 & 0.92 & -1.86 \\
\hline Fixation Duration_7 & 1.07 & 0.88 & 0.39 & 0.47 & 0.83 & -0.76 & 0.92 & -0.82 \\
\hline Fixation Duration_8 & 1.22 & 0.93 & -0.01 & 0.47 & -0.02 & -1.10 & 0.92 & -1.20 \\
\hline
\end{tabular}

Table 5: Descriptive statistics of fixations and fixation durations.

* Fixation duration unit: $\mathrm{s}$

\subsubsection{Values of luminance contrast and visual attention}

One-way repeated-measures analyses of variance were performed to discuss luminance contrast values' influences on fixations and fixation durations. The data did not pass the normality tests. The histograms, kurtosis, and skewness of each group of data suggested there was a moderate violation. We also calculated the Z-Score of kurtosis and skewness (Z-Score $< \pm 1.96$ ), as shown in Table 5. Results indicated the data approximately obeyed the normal distribution. If each group's sample size is similar, moderate violation in normality distributions will not lead to possible type I error. ANOVA is robust. Therefore, one-way repeated measures analyses of variance were still applicable.

The data met the assumption of Mauchly's Test of Sphericity $\quad\left(\chi^{2}=36.891, \quad \mathrm{df}=27, \quad \mathrm{P}=.103\right) . \quad$ One-way repeated-measures analyses of variance showed that the main effect of luminance contrast values on fixations was significant $(\mathrm{F}=3.253, \mathrm{df}=7, \mathrm{P}=0.003)$. The Post-Pairwise Comparisons explained the main effect. The fixations $(\mathrm{M}=4.71, \mathrm{SD}=3.75)$ at the luminance contrast value of 3.35 (i.e., the 7 th measurement level) was significantly less $(\mathrm{P}=0.043)$ than the fixations $(\mathrm{M}=8.13, \mathrm{SD}=3.38)$ at the luminance contrast value of 1.43 (i.e., the 3 rd measurement level), similarly, the fixations at the luminance contrast value of 3.35 was also significantly less $(\mathrm{P}=0.013)$ than the fixations $(\mathrm{M}=8.54, \mathrm{SD}=4.67)$ at the luminance contrast value of 1.11 (i.e., the $1 \mathrm{st}$ measurement level), as shown in Figure 9. Overall, the effect of luminance contrast value on fixations was statistically significant, as luminance contrast value ranged from minimum to maximum, fixations decreased significantly.
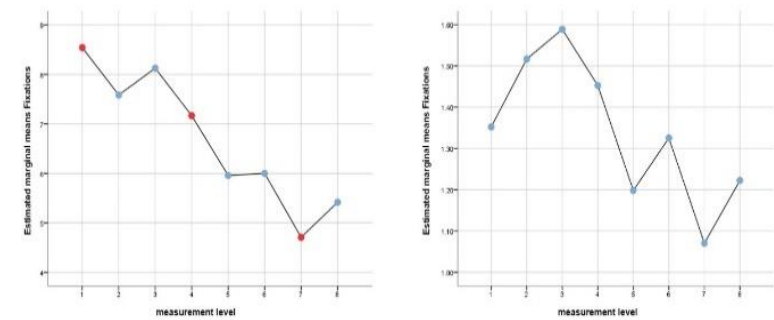

Fig 9: The effect of luminance contrast values on fixations and fixation durations (The red points reached significant differences).

The effect of luminance contrast values on fixation durations was estimated in the same way. Mauchly's Test of Sphericity failed, so the Huynh-Feldt Correction (Epsilon $(\varepsilon)=.879$ ) was adopted. It was found that no matter how the luminance contrast value changed, the fixation durations did not show any significant difference $(\mathrm{F}=1.299, \mathrm{df}=6.156, \mathrm{P}=.261)$ during the process. Although shorter fixation durations in higher luminance contrast values could be observed from descriptive statistics, that was not enough to reach the statistically significant differences. 


\subsubsection{Relations between luminance contrast and visual attention}

The Pearson correlations analyses were performed to verify the relations between the values of luminance contrast, fixations and fixation durations. The raw data distribution was far different from normality, owing to the reason one participant accepted multiple measurements in different values of luminance contrast at once. Hence the raw data needed to be transformed. Fixations and fixation durations measured in the same luminance contrast values were merged as their averages, creating the new transformed variables, mean of fixations $\left(\overline{F_{n}}\right)$ and fixation durations $\left(\overline{F_{d}}\right)$, which gather all the ocular data of participants representatively. Normality test was performed once again and succeeded.

Judging by scatterplots and the result of Pearson correlation analyses, there was a strong linear negative correlation between luminance contrast values and fixations $(r=-.801, P=.017)$. Along with the value of luminance contrast increased, fixations of participants on the sign poster decreased. Linear regression was run, resulting in a significant model $\left(\mathrm{R}^{2}=.642, \mathrm{~F}=10.770\right.$, $\mathrm{P}=.017$ ), luminance contrast value explained $64.2 \%$ of the variance in participants' fixations with moderate impact. According to the model summary output by SPSS, the linear equation is obtained as:

$$
\overline{F_{n}}=8.583-0.826 \cdot R
$$

The intercept $(\mathrm{P}<0.001)$ and the regression coefficient $(\mathrm{P}=.017)$ were both significant. The equation demonstrated that each increase in luminance contrast value by one unit reduces the mean of fixations by 0.826 (95\% CI: 1.441 0.210). When the value of luminance contrast was 1 , the predictive mean of fixations was 7.757 (95\% CI: 6.654 8.860), When the value of luminance contrast came to 3 , the predictive mean of fixations decreased to 6.106 (95\% CI: 5.227 6.984), the fitting result is shown in Figure 10.

The correlation between luminance contrast value and fixation duration was evaluated with the same method. The Pearson correlation analyses suggested a nonsignificant linear correlation $(\mathrm{r}=-.650, \mathrm{P}=.081)$, but a Spearman correlation $(\mathrm{r}=-.714, \mathrm{P}=.047)$ was negative with moderate impact. It was inferred through scatterplots that part of the reasons might come from abnormal ocular data influences influenced by extreme luminance contrast values. At the measurement level of 8 , the luminance contrast value came to 5.02, which was far beyond the reasonable range of $[1,2,3]$. In brief, the participants' fixation durations on the sign poster decreased as the value of luminance contrast increased. Although it did not fit a linear model, the correlation remained moderate and negative, as shown in Figure 11.

\section{Discussion}

In this experiment, luminance contrast was a saliency feature that distinguished the target object from the surrounding object. Affected by luminance contrast,

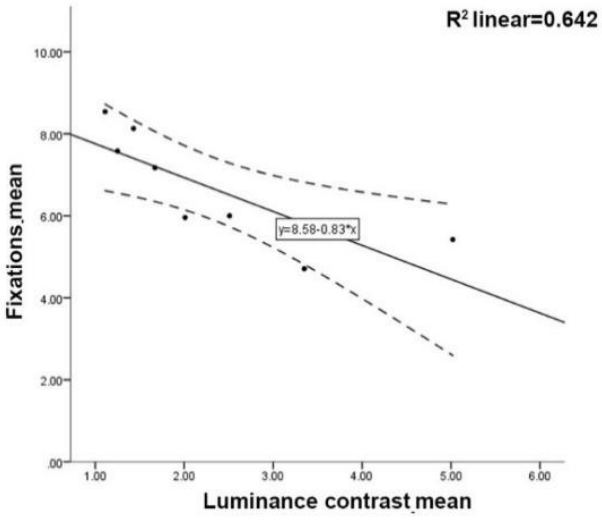

Fig 10: The correlation between luminance contrast value and fixations (Solid line is the fitting line and dotted lines represent $95 \%$ confidence interval).

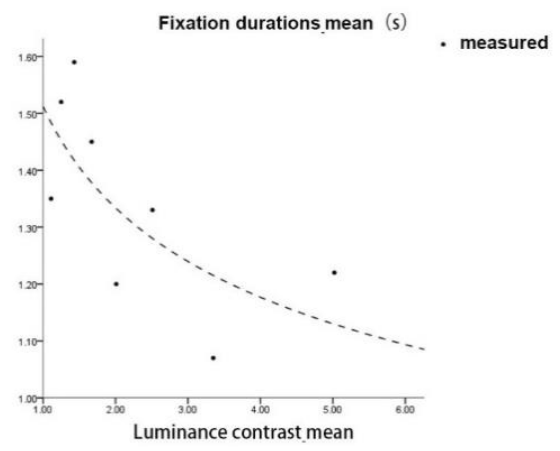

Fig 11: The correlation between luminance contrast value and fixation durations (dotted line represents negative correlation).

visual attention shows different search patterns [34] Therefore, it influenced people's information perception, which is the primary phase of wayfinding for pedestrians in metro stations [35].

The luminance contrast enhances the distinction between the sign poster and its surrounding objects, making the target more obvious. Since pedestrians consume less cognitive resources to recognize the target, they are capable of dealing with the information in a wider range. Thus, every single fixation can effectively take up information in a larger area of the visual field, all this process facilitates a global searching mode. The "global mode" is useful for identifying the overall environment by extracting contextual information, which behaves as fewer fixations and shorter fixation durations in this experiment. On the contrary, when the luminance contrast is plain, the local model of observation centered on the central fovea will hinder a wide range of perception in order to concentrate limited cognitive resources. At this time, pedestrians are more likely to be aware of signs and produce more fixations and longer fixation durations.

Zang et al. [36] researched the influences of luminance contrast and ambient lighting on visual contextual retrieval, they found longer fixation durations for search targets on screen in low luminance contrast. The results were consistent with our findings, which indicated luminance contrast led to different visual search modes. 
The study of Armougu et al. [37] showed that longer fixation durations might be related to a higher cognitive load, pedestrians tended to perform this behavior in an unfamiliar metro station environment because they needed to mobilize attention resources with efforts to stay alert and obtain what they demanded from massive information. While in cognitive load, pedestrians required fewer attention resources to check only for the correct information, which led to shorter fixation durations.

Lin et al. [38] found a highly positive correlation between subjective discomfort caused by luminance contrast and eye movement speed, which was consistent with our findings, that is, in the higher luminance contrast, the eye kept moving with a stronger feeling of glare. Pakkert et al. [39] concluded in their study that presenting a small number of lights yielded improvement would not show in the task performance. The author said that it was hard to determine the specific effect of the glare caused by extreme luminance contrast on the observer's eye because the human body could make a "strategic adaptation", our findings partially confirmed this conclusion. In the "information perception" phase of wayfinding, higher luminance contrast can facilitate the efficient "global mode", but a deep feeling of disturbing is aroused in the top-down mechanism's intervention. Conversely, the "focal mode" induced by lower luminance contrast consumes more cognitive resources. However, it results in a higher subjective comfort in the intervention of the topdown mechanism, and it is implied that the bottom-up mechanism and the top-down mechanism may make adjustments in two opposite directions to reach a "strategic balance" in the final task performance.

The current lighting standards do not clearly define luminance and contrast, lacking consideration for pedestrian's visual perception. This research provides parameters and formulas that can be used directly, from which the related professionals engaged in planning, implementation, and maintenance of lighting in large indoor public transportation spaces can benefit. One point to stress here is that the method developed in this research combined virtual reality with eye tracking, establishing a high-fidelity simulated lighting environment in static or dynamic states, rare in previous studies. Firstly, the on-site measurements were implemented to restore the panoramic images and videos of the actual scene, the simulation software was utilized to control lighting conditions accurately. Then, eye-tracking data were collected to be analyzed at the fixed time of interest. Simultaneously, participants' natural gaze behavior was recorded between milliseconds to reveal the slight visual differences. All of these significantly improved the experimental accuracy in the virtual environment $[20,24,31,40]$. Advanced lighting simulation with eye-tracking is of great importance for some special situations, such as traffic safety in driving and pedestrians $[19,41,42,43]$. The method's advantages could prove to be beneficial for visual perception and human factors research.

The luminance contrast between the sign and the surrounding object does affect pedestrians' visual perception, but whether wayfinding performance will be influenced is still unknown. At this stage, limited by the limited of VR, we have difficulty in restoring the interactive behavior of pedestrians in the real world, only discontinuous movements between several fixed viewpoints were achieved, which is far different from the actual situation. It is confirmed that the luminance of the sign and its surrounding objects should be under reasonable control when lighting the guide system for large indoor public transportation spaces. If pedestrians are expected to browse and locate assorted information in the space quickly, there is supposed to be a large luminance contrast between signs and other objects, if pedestrians are expected to focus on the critical navigation information, it is supposed to set similar luminance for signs and other objects. In practical applications, low luminance contrast at wayfinding decision points is recommended, high luminance contrast during periods of heavy traffic, or at the points where wayfinding task is accessible (e.g. straight corridor) is also advised. Besides, according to field research, the phenomenon of excessive lighting in Chinese metro stations is severely common nowadays. In fact, non-luminous signs at low cost like posters make much sense. If signs and their surrounding advertisement boards are both luminous, one of them will require significantly higher luminance to be distinguished from the other which results in more energy consumption, cautious attitude should be taken when adding lighting to signs and its surrounding objects.

\section{Conclusion}

These results have shown that luminance contrast is a saliency feature distinguishing visual targets and surrounding objects. On condition with luminance contrast, pedestrians observed the signs with fewer fixations and fixation durations, both of which decreased as luminance contrast increased, it depended on the "global mode" dominated by bottom-up mechanisms in high luminance contrast. Compared with the performances on conditions without luminance contrast, participants were disturbed more severely in visual perception on condition with luminance contrast, which was explained as the "strategic adaptation" of the top-down mechanisms and bottom-up mechanisms in two opposite directions

The method of combining lighting simulation with eye-tracking made it gain precise ocular data in the static or dynamic panorama based on a virtual lighting environment, which provides new insight into the lighting design of ample indoor public transportation space. The effect of luminance contrast on pedestrian's visual perception supports that luminance contrast between sign and surrounding object should be reasonably controlled according to different wayfinding situations to ensure a beneficial relation and difference in pedestrian's eye.

\section{Declaration of competing interests}

The authors declared that they have no known competing financial interests or personal relationships that could have appeared to influence the work reported in this paper. 


\section{Acknowledgments}

This work is supported by the National Natural Science Foundation of China (51808232), Social Science Foundation of the Fujian Province, China (FJ2021B178).

\section{References}

[1] Fotios, S., \& Gibbons, R.(2018). Road lighting research for drivers and pedestrians: The basis of luminance and illuminance recommendations. Lighting Research \& Technology, 50(1), 154-186. https://doi.org/10.1177/1477153517739055.

[2] Kruisselbrink, T., Dangol, R., \& Rosemann, A. (2018). Photometric measurements of lighting quality: An overview. Building and Environment, 138, 42-52.

https://doi.org/10.1016/j.buildenv.2018.04.028.

[3] International Commission on illumination. LIGHTING OF WORK PLACES - PART 1: INDOOR. ISO 8995-1:2002(E)/CIE S 008/E:2001. https://cie.co.at/publications/lighting-work-placespart-1-indoor.

[4] Hamedani, Z., Solgi, E., Hine, T., \& Skates, H.(2020). Revealing the relationships between luminous environment characteristics and physiological, ocular and performance measures: An experimental study. Building and Environment, 172, 106702.

https://doi.org/10.1016/j.buildenv.2020.106702.

[5] Amundadottir, M. L., Rockcastle, S., Khanie, M.S., \& Andersen, M. (2017). A human-centric approach to assess daylight in buildings for non-visual health potential, visual interest and gaze behavior. Building and Environment, 113, 5-21.

https://doi.org/10.1016/j.buildenv.2016.09.033.

[6] Sarey Khanie, M., Stoll, J., Einhäuser, W., Wienold, J., \& Andersen, M. (2015). Gaze-driven approach for estimating luminance values in the field of view for discomfort assessments (No. CONF).

[7] Hamedani, Z., Solgi, E., Skates, H., Hine, T., Fernando, R., Lyons, J., \& Dupre, K. (2019). Visual discomfort and glare assessment in office environments: A review of light-induced physiological and perceptual responses. Buildingand Environment, 153, 267-280. https://doi.org/10.1016/j.buildenv.2019.02.035.

[8] Le Meur, O., \& Baccino, T. (2013). Methods for comparing scanpaths and saliency maps: strengths and weaknesses. Behavior research methods, 45(1), 251-266. https://doi.org/10.3758/s13428-012-0226-9

[9] Lund, H., (2016). Eye tracking in library and information science: a literature review. Library $\mathrm{Hi}$ Tech 34, 585-614. https://doi.org/10.1108/LHT-07-2016-0085

[10] Singh, J., \& Modi, N. (2019). Use of information modelling techniques to understand researchtrends in eye gaze estimation methods: An automated review. Heliyon, 5(12), e03033.

https://doi.org/10.1016/j.heliyon.2019.e03033.
[11] Borji, A., \& Itti, L. (2012). State-of-the-art in visual attention modeling. IEEE transactions on pattern analysis and machine intelligence, 35(1), 185-207. https://doi.org/10.1109/TPAMI.2012.89

[12] Charles, R. L., \& Nixon, J. (2019). Measuring mental workload using physiological measures: A systematic review. Applied ergonomics, 74, 221232. https://doi.org/10.1016/j.apergo.2018.08.028.

[13] Cosma, G., Ronchi, E., \& Nilsson, D. (2016). Wayfinding lighting systems for rail tunnel evacuation: A virtual reality experiment with Oculus Rift ${ }^{\circledR}$. Journal of Transportation Safety \& Security, 8(sup1), 101117. https://doi.org/10.1080/19439962.2015.1046621

[14] Tang, M., \& Auffrey, C. (2018). Advanced digital tools for updating overcrowded rail stations:using eye tracking, virtual reality, and crowd simulation to support design decision-making. Urban Rail Transit, 4(4), 249-256. https://doi.org/10.1007/s40864-018-0096-2

[15] Wu Z., (2018) Empirical Study on the Optimization Strategy of Subject Metro Design Based on Virtual Reality. Informatica, 42(3). https://doi.org/10.31449/inf.v42i3.2424.

[16] Ai X, Wu Z, Guo T, et al., (2021). The effect of visual attention on stereoscopic lighting of museum ceramic exhibits: A virtual environment mixed with eye-tracking.Informatica,45(5). https://doi.org/10.31449/inf.v45i5.3454.

[17] Schrom-Feiertag, H., Settgast, V., \& Seer, S. (2017). Evaluation of indoor guidance systems using eye tracking in an immersive virtual environment. Spatial Cognition \& Computation, 17(1-2), 163-183. https://doi.org/10.1080/13875868.2016.1228654.

[18] Suzer, O. K., Olgunturk, N., \& Guvenc, D. (2018). The effects of correlated colour temper- ature on wayfinding: A study in a virtual airport environment. Displays, 51, 9-19. https://doi.org/10.1016/j.displa.2018.01.003.

[19] Chamilothori, K., Chinazzo, G., Rodrigues, J., DanGlauser, E. S., Wienold, J., \& Andersen, M. (2019). Subjective and physiological responses to façade and sunlight pattern geometry in virtual reality. Building and Environment, 150, 144-155. https://doi.org/10.1016/j.buildenv.2019.01.009.

[20] Berton, F., Hoyet, L., Olivier, A. H., Bruneau, J., Le Meur, O., \& Pettré, J. (2020). Eye-gaze activity in crowds: impact of virtual reality and density. In 2020 IEEE Conference onVirtual Reality and 3D User Interfaces (VR) (pp. 322-331). IEEE. https://doi.org/10.1109/VR46266.2020.00052

[21] Zahabi, M., Machado, P., Lau, M.Y., Deng, Y., Pankok, C., Jr., Hummer, J., Rasdorf, W., Kaber, D.B., (2017). Driver performance and attention allocation inuse of logo signs on freeway exit ramps. Applied ergonomics 65, 70-80. https://doi.org/10.1016/j.apergo.2017.06.001

[22] Jones, N. L., \& Reinhart, C. F. (2017). Experimental validation of ray tracing as a means of image-based visual discomfort prediction. 
Building and Environment, 113, 131-150. https://doi.org/10.1016/j.buildenv.2016.08.023.

[23] Mahić, A., Galicinao, K., \& Van Den Wymelenberg, K. (2017). A pilot daylighting field stu-dy: Testing the usefulness of laboratory-derived luminancebased metrics for building design and control. Building and Environment, 113, 78-91. https://doi.org/10.1016/j.buildenv.2016.11.024.

[24] Chen, Y., Cui, Z., \& Hao, L. (2019). Virtual reality in lighting research: Comparing physical and virtual lighting environments. Lighting Res-earch \& Technology, 51(6), 820-837. https://doi.org/10.1177/1477153518825387.

[25] German Institute for Standardization. Artificial lighting - Part 8: Workplace luminaries Requirements, recommendations and proofing. DIN 5035-8 : 2007-07. https://www.beuth.de/de/norm/ din-5035-8/98019763.

[26] Pedersen, E., \& Johansson, M. (2018). Dynamic pedestrian lighting: Effects on walking speed, legibility and environmental perception. Lighting Research \& Technology, 50(4), 522-536. https://doi.org/10.1177/1477153516684544.

[27] Kim, I. T., Choi, A. S., \& Sung, M. K. (2018). Accuracy evaluation of a calculation tool based on the spectral colour property of indoorluminous environments. Building and Environ-ment, 139, 157 169. https://doi.org/10.1016/j.buildenv.2018.05.028.

[28] Murdoch, M. J., Stokkermans, M. G., \& Lambooij, M. (2015). Towards perceptual accuracy in 3D visualizations of illuminated indoor environments. Journal of Solid State Lighting, 2(1), 1-19. https:// doi.org /10.1186/s40539-015-0029-6.

[29] Moscoso, C., Matusiak, B., Svensson, U. P., \& Orleanski, K. (2015). Analysis of stereoscopic images as a new method for daylighting studies. ACM Transactions on Applied Perception (TAP), 11(4), 1-13 https://doi.org/10.1145/2665078.

[30] Hidayetoglu, M. L., Yildirim, K., \& Akalin, A.(2012). The effects of color and light on in- door wayfinding and the evaluation of the per- ceived environment. Journal of environmental psychology, 32(1), 50-58. https://doi.org/10.1016/j.jenvp.2011.09.001.

[31] Bian, Y., \& Luo, T. (2017). Investigation of visual comfort metrics from subjective res- ponses in China: A study in offices with day- light. Building and Environment, 123, 661-671. https://doi.org/10.1016/j.buildenv.2017.07.035.

[32] Cheng, T. J., Yang, B., Holloway, C., \& Tyler, N. (2018). Effect of environmental factors on how older pedestrians detect an upcoming step. Lighting Research \& Technology, 50(3), 405-415. https://doi.org/10.1177/1477153516669968.

[33] Fotios, S., Uttley, J., Cheal, C., \& Hara, N. (2015). Using eye-tracking to identify pedestrians'critical visual tasks, Part 1. Dual task approach.Lighting research \& technology, 47(2), 133-148. https://doi.org/10.1177/1477153514522472.

[34] De Boer, J., (1967). Public lighting. Cleaver-Hume.
[35] Gellatly, A. W., \& Weintraub, D. J. (1990). User reconfigurations of the de Boer rating scale for discomfort glare. University of Michigan, Ann Arbor, Transportation Research Institute.

[36] Shiferaw, B., Downey, L., \& Crewther, D. (2019). A review of gaze entropy as a measure of visual scanning efficiency. Neuroscience \& Bio-behavioral Reviews, 96, 353-366. https://doi.org/10.1016/j.neubiorev.2018.12.007.

[37] Arthur, P., Passini, R., (1992). Wayfinding: people, signs, and architecture.

[38] Zang, X., Huang, L., Zhu, X., Müller, H. J., \& Shi, Z. (2020). Influences of luminance contrast and ambient lighting on visual context learning and retrieval. Attention, Perception, \& Psychophysics, 82(8), 4007-4024. https://10.3758/s13414-020-02106-y.

[39] Armougum, A., Gaston-Bellegarde, A., Joie-La Marle, C., \& Piolino, P. (2020). Physiological investigation of cognitive load in real-life train travelers during information processing. AppliedErgonomics, 89, 103180. https://doi.org/10.1016/j.apergo.2020.103180.

[40] Lin, Y., Fotios, S., Wei, M., Liu, Y., Guo, W., \& Sun, Y. (2015). Eye movement and pupil size constriction under discomfort glare. Investigative ophthalmology \& visual science, 56(3), 1649-1656. https://doi.org/10.1167/iovs.14-15963.

[41] Pakkert, M., Rosemann, A. L., van Duijnhoven, J., \& Donners, M. A. (2018). Glare quantification for indoor volleyball. Building and Environment, 143, 48-58. https://doi.org/10.1016/j.buildenv.2018.06.053.

[42] Higuera-Trujillo, J.L., Lopez-Tarruella Maldonado, J., Llinares Millan, C., (2017). Psychological and physiological human responses to simulated and real environments: A comparison between Photographs, 360 degrees Panoramas, and Virtual Reality. Applied ergonomics 65, 398-409. https://doi.org/10.1016/j.apergo.2017.05.006

[43] Deb, S., Carruth, D.W., Sween, R., Strawderman, L., Garrison, T.M., (2017). Efficacy of virtual reality in pedestrian safety research. Applied ergonomics 65, 449-460.

https://doi.org/10.1016/j.apergo.2017.03.007 